\title{
TEMPORALIDADY CONTAMINACIÓN COMO CLAVES PARA INTERPRETAR LA BIODIVERSIDAD DE MACROINVERTEBRADOS EN UN ARROYO MEDITERRANEO (Riera de Sant Cugat, Barcelona)
}

\author{
N. Bonada, M. Rieradevall y N. Prat \\ Departament d'Ecologia. Facultat de Biologia. Universitat de Barcelona. \\ Avda. Diagonal, 645. 08028 Barcelona. Spain.
}

\section{RESUMEN}

En Octubre de 1995 se estudiaron los macroinvertebrados acuaticos en varias localidades de la cuenca de la Riera de Sant Cugat (Barcelona) situadas dentro y fuera del Parque Metropolitano de Collserola y, se relacionaron con la calidad y la temporalidad de las aguas.

Gran parte del recorrido de los cauces estaba seco en el momento de estudio. Algunas localidades, situadas en los límites del Parque, poseian flujo permanente pero con elevados signos de contaminacibn. Dentro del Parque se encontraron algunas zonas con caudal permanente, aunque reducido (desde menos de $11 / \mathrm{s}$ hasta $32 \mathrm{l} / \mathrm{s}$ ), y zonas con flujo intermitente, además de los que estaban secos.

En las quince localidades muestreadas se encontraron un total de 65 taxones distintos; la mayor riqueza en una sola estación fue de 33 y se encontraron tanto especies de zonas redfilas (simúlidos o algunos efemerópteros) como aquellas más propias de aguas lénticas como hemipteros, odonatos o algunos coleópteros. Mediante un análisis de ordenación en el espacio se agruparon las estaciones y se ordenaron en función de su flujo y del grado de polucibn, diferenciándose claramente las estaciones externas al parque (con flujo moderado y cierta contaminacidn) de aquellas situadas en el interior del parque y, entre Cstas, las de flujo permanente respecto a las encharcadas. Se analizaron las diferencias entre estos cuatro grupos según los tipos de organismos existentes y su estrategia trófica.

A pesar de que muchos puntos de muestreo presentaban poca agua, se refuerza la idea de la importancia de mantener los flujos mínimos de estos rios para conservar la biodiversidad.

Palabras clave: temporalidad, contaminacidn, calidad del agua, macroinvertebrados, diversidad, niveles tróficos, zonas protegidas

\begin{abstract}
In October 199.5, the aquatic macroinvertebrate community of the Sant Cugat stream (Barcelona) was examined at various sampling points. Several streams were located inside of the Collserola Metropolitan Park while the main section was in the border area. The community was related to the pollution and theflow seasonality of the stream.

Most of the sampling points, located close to the edge of the Park, had permanentflow but with high pollution. On the other hand, inside the Park there were localities with lowflow (from $<1 l / s$ to $32 l / s$ ), others with intermittentflow, and several without water:

In the fifteen localities sampled a total of 6.5 taxa werefound; the highest species richnessfound was 33 taxa of reophilus species (such us simulidae and ephemeroptera) as well as lentic species such as hemiptera, odonata and some coleoptera. Using an ordenation analysis, sampling points were grouped according to theirflow and pollution level. The localities outside the Park (with moderateflow and high pollution) are clustered apart from those inside the Park, where sampling points with permanent water flow and intermittent waterflow are clearly differentiated. The differences between thefour groups of sampling points with distinct macroinvertebrate community also show differences in the dominance of trophic groups, with collector-gatherers dominant in polluted areas.
\end{abstract}

In temporary streams, the presence of permanent streams (even with very lowflow or only pools) is a keyfactor to maintain biodiversity.

Keywords: seasonality, pollution, water quality, macroinveterbrates, diversity, trophic levels, protected zones

Limnética 18:81-90 (2000)

(C) Asociación Espaiiola de Limnologia, Madrid. Spain 


\section{INTRODUCCIÓN}

Los rios de régimen mediterrhneo poseen grandes fluctuaciones de caudal (Sabater et al., 1993; Giudicelli et al., 1985; Sabater et al., 1995), hasta el punto de que en algunas épocas del aiio pueden hallarse total o parcialmente sin agua (Ferreras-Romero \& García-Rojas, 1995; Coimbra et al., 1996), siendo un caso extremo las ramblas del sureste peninsular (Molina et al., 1994). Ademhs, se ven frecuentemente alterados por las derivaciones de agua y la contaminación (Prat et al., 1985, 1996 y 1997; Prenda \& Gallardo-Mayenco, 1996; Coimbra et al., 1996; Maamri et al., 1997), 10 que unido a la temporalidad propia de su régimen hidrológico supone la existencia de unos ecosistemas peculiares (Moreno et al., 1996, 1997). Las crecidas pueden ser un factor importante en la limitación de su biodiversidad, aunque los rios mediterrhneos muestran una capacidad importante de recuperación y pueden mantener comunidades relativamente complejas (Vidal-Abarca et al., 1991; Ortega et al., 1991).

La dinhmica y el funcionamiento de las comunidades de macroinvertebrados en este tipo de rios y en Espaiia ha sido relativamente poco estudiada, aunque existen cada vez más datos de diferentes regiones del levante peninsular (Prat et al., 1985; Puig et al., 1986; Vidal-Abarca et al., 1991, 1992; Ortega et al., 1991; Gallardo, 1993; Prenda \& Gallardo-Mayenco, 1996; Martínez-López et al., 1996; Moreno, 1996; Dominguez et al., 1997; Del Moral et al., 1997). Por otro lado, el efecto de la protección de espacios en áreas naturales y/o periurbanas sobre la fauna macroinvertebrada de los ecosistemas acuhticos que incluyen, ha sido poco o nada estudiado (Rieradevall et al., en prensa).

El objetivo del presente estudio es caracterizar, desde el punto de vista de su composición y estructura trófica, la comunidad de macroinvertebrados acuáticos de los arroyos que conforman la cuenca de la Riera de Sant Cugat y su relación con distintos tipos de perturbaciones, ya sean reducciones de caudal o contaminación por vertidos, en zonas protegidas $\mathbf{y}$ no protegidas.

\section{AREA DE ESTUDIO}

La Sierra de Collserola (Parque Forestal desde 1976 y actualmente Parque Metropolitano) con 6.500 Ha esth situada en la vertiente noroeste de Barcelona y limita al este con el Rio Besós y al oeste con el Rio Llobregat. La Riera de Sant Cugat, tributaria del Rio Ripoll (afluente del Be\&), bordea la zona norte del Parque y sus afluentes del margen derecho discurren dentro del mismo. La parte media y baja de la cuenca transcurre a través de pueblos importantes, circundantes al Parque, como Sant Cugat del Vallés y Cerdanyola del Vallés. En algunos tramos dentro del Parque los cauces se encuentran alterados por impactos urbanisticos más o menos importantes.

En general, los cauces poseen poca pendiente como consecuencia de la reducida altitud del Parque. Sus lechos esthn constituidos por arenas, gravas y piedras de canto rodado a veces con materiales artificiales, en zonas urbanizadas, $\mathrm{o}$ con hojarasca en zonas muy cerradas por la vegetación de ribera. Dentro del Parque, la vegetación de ribera suele ser importante en algunos de los arroyos, proporcionando elevados niveles de cobertura. Las especies predominantes son chopos, olmos, encinas y pinos, junto a una abundante vegetación arbustiva. En los tramos que rodean el Parque, o los que discurren cerca de Areas urbanizadas, las riberas esthn bastante degradadas con presencia de cultivos y vegetación ruderal nitrófila. El clima de la zona es mediterrhneo con abundantes precipitaciones torrenciales, principalmente en otoiio. El sustrato silicico (pizarras) no permite la presencia de grandes acuiferos, por 10 que la mayoria de torrentes poseen flujo intermitente y en los que mantienen un caudal permanente, el flujo es reducido.

\section{METODOLOGÍA}

Se estudiaron, en una unica ocasión (Octubre de 1995), 22 localidades de la cuenca de la Riera de Sant Cugat (Fig. 1), 11 pertenecientes al Parque y 11 situados fuera pero cerca de sus limites. De estas 22 localidades, solamente 15 fueron mues- 
treadas ya que el resto o bien estaban secas, o, tras una primera exploración, se comprobó que no presentaban fauna macroinvertebrada debido a que eran pozas de agua de lluvia (estaciones 16 y 17) o eran pozas aisladas muy contaminadas en el momento del muestreo (estación 1).

En cada punto de muestreo, se realizó una caracterización visual del estado de conservación de la cuenca y de la vegetación de ribera. Ade- más se hizo una descripción del hábitat acuático, considerando tipo de substrato y microflora bentónica. También se determinaron "in situ" el caudal, la temperatura, la conductividad y el oxígeno disuelto mediante los correspondientes aparatos de campo (los resultados se pueden ver en Bonada et al., 2000).

El macrobentos se capturó mediante una red de $250 \mu \mathrm{m}$ de malla procurando muestrear todos los

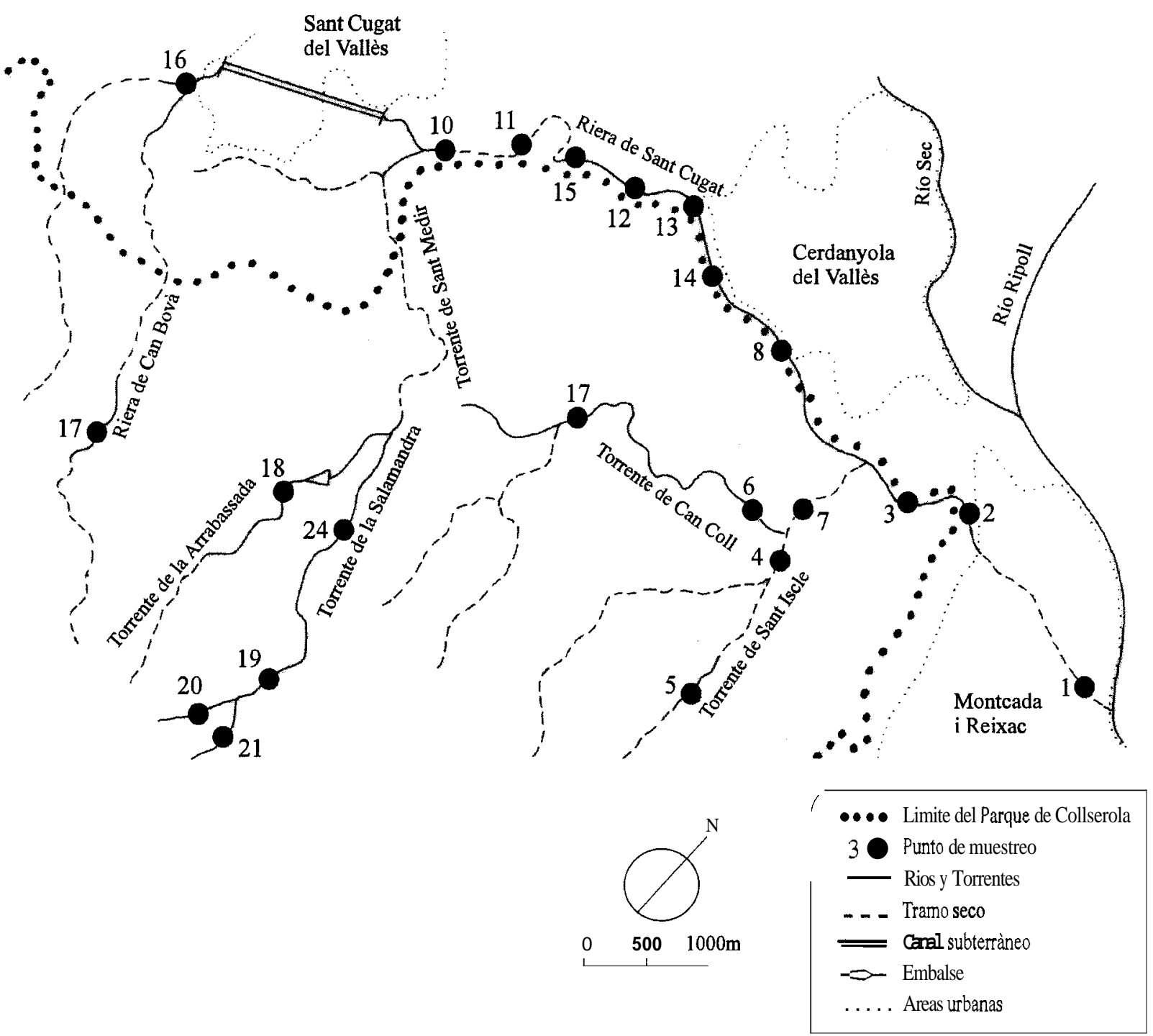

Figura 1. Mapa de localidades muestradaa. Ver significado de los símbolos en la leyenda. Location o the sampling sires. See legendfor explanation. 
substratos presentes con el mismo esfuerzo de muestreo. Las muestras son, pues, semicuantitativas y asi se han tratado. Se fijaron en formol al $4 \%$ para su posterior separación en el laboratorio, donde se separaron y se identificaron los organismos. La identificación se realizó hasta el nivel taxonomico mas bajo posible (género o especie), excepto para algunos dipteros o bien cuando los individuos pertenecían a estadios muy pequeiios. La determinación de la calidad biolbgica de las aguas se realizó mediante la aplicación de dos indices biológicos, el BMWP' (Alba-Tercedor \& Sanchez-Ortega, 1988) y el BILL (Prat et al., 1985; 1996).

La caracterización de la comunidad de macroinvertebrados se realizó mediante el estudio de la similaridad entre muestras aplicando de forma exploratoria varios indices y utilizando varios algoritmos de agrupacion aglomerativa. Se presentan los resultados obtenidos con el índice de similaridad de Steinhaus y mediante la agrupación UPGMA (unweighted pair-group method using arithmetic averages) por ser los que mostraron agrupaciones más claras de las comunidades. Finalmente, se analizó la estmctura de la matriz de similaridades entre muestras en relación a su posición en el espacio geografico real mediante el método de "constrained-clustering". Con el cluster resultante de este método se dibujó un mapa de coordenadas geograficas con los distintos niveles de agrupación definidos por el método. Para cada una de las sucesivas fases del análisis estadistico de los datos se utilizaron los subprogramas SIMIL, CLUSTER y BIOGEO (Legendre \& Vaudor, 1991).

\section{RESULTADOS}

\section{Caracteristicasfísicas y quimicas}

Un $58,65 \%$ de los cursos de agua de la cuenca de la riera de Sant Cugat se hallaban secos en el momento de muestreo. De las 22 estaciones prospectadas, cinco estaban secas (localidades 1, 4, 7, 10 y 11 ), ocho presentaron un caudal inferior a 1 $1 / \mathrm{s}$ (puntos $2,5,6,9,16,17,20$ y 21 ) mientras que las restantes tenian un caudal de entre 7,5 1/s y 32 $1 / \mathrm{s}$. La mayoría de las estaciones presentaban concentraciones de oxígeno elevadas (entre 5,5 y $17,6 \mathrm{mg} / \mathrm{l})$, ya que la riera no recibia grandes volumenes de aguas residuales (las cuales se encontraban canalizadas hacia la depuradora de Montcada i Reixac) y el agua que circulaba normalmente, provenia de fuentes o de pequeñas aportaciones no conectadas al sistema general de colectores.

Las localidades situadas en el interior del Parque presentaron valores de saturación de oxígeno inferiores al $100 \%$, como consecuencia de la densa cobertura del bosque que impide la llegada de la luz y el desarrollo de la comunidad algal. Esto no ocurre en los puntos externos al Parque, donde la ausencia de cobertura riparia permite la presencia de comunidades importantes de diatomeas y cianófitos, lo que origina valores de sobresaturación de oxigeno en alguna localidades (estaciones 2 y 3 ).

\section{Composición de la comunidad de macroin- vertebrados y calidad de las aguas}

En total se han identificado 65 taxones. De estos, las familias mejor representadas por su frecuencia y abundancia son las de los Quironómidos, Simulidos y Gammáridos. Las zonas protegidas por el Parque, con cierto caudal (estaciones $19 \mathrm{y}$ 24), presentan una mayor variedad de grupos que las localidades con menor flujo (20 y 21). Las localidades circundantes al Parque (12, 13 y 14) tienen -a pesar de poseer un caudal mayorcomunidades poco diversas y mas propias de zonas contaminadas con mayor abundancia de quironómidos, simúlidos y oligoquetos. La autodepuración se refleja aguas abajo de estos puntos, en un aumento de la diversidad (puntos 8 y 3). El listado completo de los organismos encontrados se puede consultar en Bonada et al. (2000).

La determinacion a nivel especifico de algunos grupos revela que algunas de las especies presentes son las propias de cursos temporales o intermitentes con condiciones lénticas buena parte del aiio (Electrogena lateralis, Caenis rivulorum), mientras que otras son propias de aguas relativa- 
mente frias y permanentes (Prosimulium hirtipes y Simulium (N.) vernum) como sucede en las localidades 19y 24, situadas dentro del parque. Por su parte Simulium (Eusimulium) sp. es caracteristico de zonas mas cálidas y eutrofizadas, donde también se encuentran ejemplares de Cricotopus (C.) gr. bicinctus, C. (I.) gr. sylvestris y Chironomus $c \$$ luridus, propias de aguas con elevadas concentraciones de materia organica, y halladas en las localidades situadas fuera del Parque.

Un táxon muy bien representado es el gammárido Echinogammarus longisetosus, muy frecuente en las localidades dentro del Parque con abundante hojarasca, especialmente en la estacion 6. La presencia de una unica familia de plecóptero (Nemouridae), pocos efemerópteros y pocos tricópteros muestra las limitaciones del sistema para sustentar una variada comunidad de organismos reófilos y su marcado carácter temporal.

Los indices de calidad BMWP' y BILL (Tabla 1), muestran que las localidades situadas fuera del Parque poseen niveles de calidad muy bajos. En cambio, las localidades situadas en el interior del Parque tienen buen nivel de calidad, excepto los puntos 9, 20 y 21. La estación 9, situada en el Torrent de Can Coll recibe aportes puntuales de aguas residuales que reducen su calidad (nivel III en el BMWP'). No obstante, a poca distancia se vuelve a alcanzar una buena calidad (estación 6), hecho que se constata con una recuperacion de los niveles de oxigeno (de $5,72 \mathrm{mg} / 1$ en la estación 9 a 7,42 mg/l en la 6) y un aumento de la riqueza taxonomica y de los indices de calidad aplicados (el BMWP' pasa de calidad III a I). En las localidades 20 y 21, si bien el indice BMWP' alcanza valores bajos, indicando contaminación, los organismos presentes fueron propios de aguas limpias (como E. longisetosus)

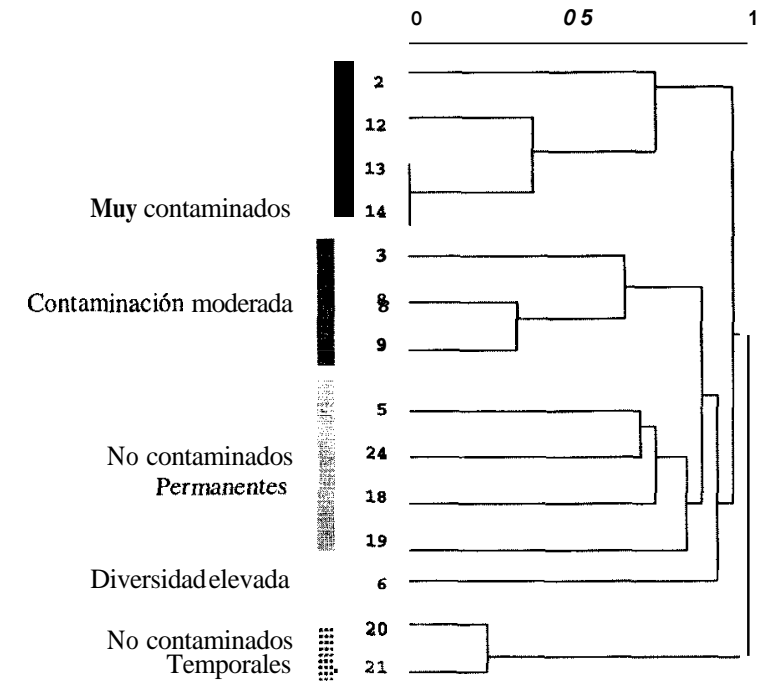

Figura 2. Dendrograma de la agrupación de las localidades segun su similaridad usando el programa CLUSTER. A las distintas agrupaciones halladas se les ha asociado un grado de contaminación y temporalidad. El dendrograma es significativo, con un $r=0,97$. Dendrogram derived from sample similarity matrix using the CLUSTER program. An ecological definition was assigned to each group. The dendrogram is significative, with $r=0.97$.

aunque poco abundantes debido a que el reducido caudal no permite mantener un elevado numero de individuos.

\section{Comparación de localidades segun su grado de similitud}

El grado de contaminación y de caracteristicas de temporalidad del caudal son importantes en la definición de las distintas comunidades de macroinvertebrados (Fig. 2). Un primer grupo aparece diferenciando las estaciones mas temporales (estaciones 20 y 21) de las permanentes. En segundo lugar, las estaciones se agrupan siguiendo

Tabla 1. Valores del BILL y rangos del índice BMWP' segun ALBA-TERCEDOR y SANCHEZ-ORTEGA (1988)en las localidades mues treadas. BILL values and BMWP' ranks according to ALBA-TERCEDOR and SANCHEZ-ORTEGA (1988)at euch sampling site.

\begin{tabular}{|c|c|c|c|c|c|c|c|c|c|c|c|c|c|c|c|}
\hline & \multicolumn{6}{|c|}{ FUERA DEL PARQUE } & \multicolumn{9}{|c|}{ DENTRO DEL PARQUE } \\
\hline & 2 & 3 & 8 & 12 & 13 & 14 & 5 & 6 & 9 & 17 & 18 & 19 & 20 & 21 & 24 \\
\hline BMWP' & II & IV & III & $\mathrm{V}$ & IV & IV & III & I & III & II & I1 & I & IV & IV & II \\
\hline BILL & 3 & 6 & 4 & 0 & 2 & 3 & 6 & 10 & 6 & 5 & 9 & 10 & 4 & 4 & 8 \\
\hline
\end{tabular}


un gradiente de contaminación, desde las no contaminadas a las muy contarninadas, pasando por estaciones de contaminación moderada. El análisis de la matriz de similaridad con constricción espacial (coordenadas geográficas) revela que las agrupaciones se situan en un gradiente altitudinal, desde las zonas de cotas más elevadas a las más bajas (Fig. 3), lo que pone de relieve la importancia de la zona protegida por el parque y en especial la importancia de las Areas de cabecera de los rios como Areas con fauna relativamente diferente y a proteger. Como las agrupaciones son las mismas en ambos análisis los comentarios pertinentes se realizaran en adelante de manera combinada. Asi, las estaciones del curso principal que se situan más allá del límite del Parque (2, 12,13 y 14) quedan agrupadas (gmpo "Muy contaminados"), ya que todas poseen valores bajos de los indices biológicos calculados (nivel $\mathrm{V}$ de BMWP' o de BILL). El numero de taxones encontrados en este grupo es muy bajo (de 1 a 4). Un segundo grupo ("Contaminación moderada") es el constituido por los puntos 3,8 y 9 que poseen contaminación moderada (III 6 IV BMWP' y 466 de valor de BILL) con flujo constante y número de taxones intermedio (entre 6 y 12).

Las localidades no contarninadas, situadas en el interior del parque, se agrupan según su flujo

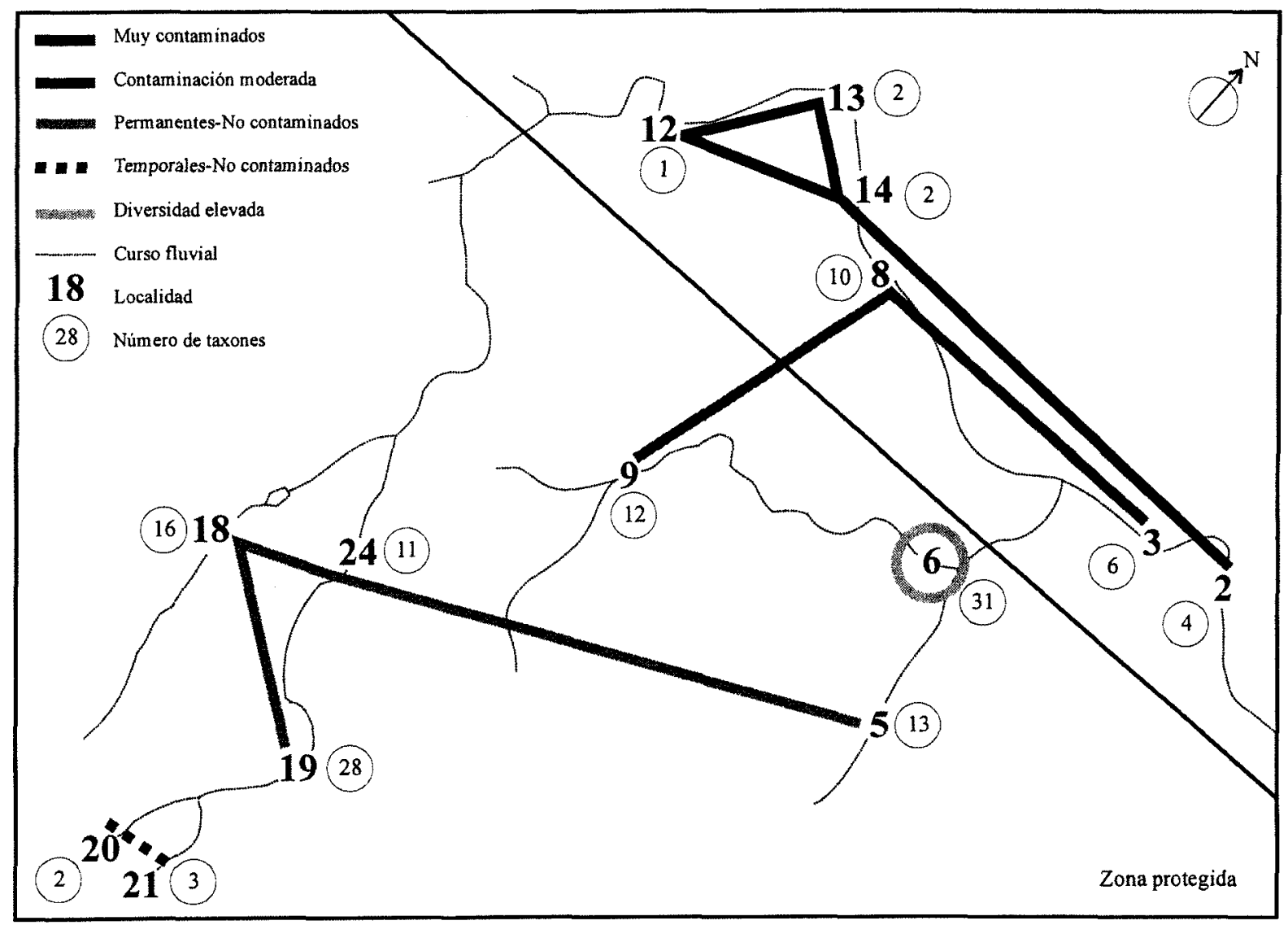

Figura 3. Proyeccidn espacial sobre coordenadas geograficas (programa BIOGEO) de los grupos de localidades mostrados en el dendrograma de la Figura 2. La línea recta representa los limites de protección del parque. Los valores dentro de los circulos indican el número de taxones hallados en cada estación. Spatial projection using geographical coordinates (BIOGEO program) ojthe groups of sampling sites arising from the cluster: Different shading lines refer to the same groups as in Figure 2. The straight line marks the Park protection boundary. The values inside the circles are the number of taxafound. 
("No contaminados-Permanentes" y "No contaminados-Temporales"). En el segundo grupo, tenemos los tramos de cabecera (20 y 21) con muy bajo caudal, 10 que supone bajo número de individuos y variedad especifica (sólo 2 ó 3 especies). Las zonas con aguas permanentes y fluyentes no contaminadas $(5,24,18$ y 19) son las más diversificadas, con mayor riqueza específica (entre 11 y 28 taxones distintos) y con numero elevado de individuos, así como especies indicadoras de buena calidad de las aguas. Por otra parte, la estación 6, a pesar de tratarse de una poza con un flujo de renovación bajo, posee elevada abundancia y diversidad de individuos, con especial atención al gammárido Echinogammarus longisetosus, cuya abundancia se maximiza por la cobertura del bosque en este punto que aporta gran cantidad de materia orgánica para la alimentación del crustaceo.

\section{Estructura trófica de las comunidades}

La estructura trofica de cada localidad se ha analizado de acuerdo con el tipo de alimentación que cada táxon tiene asignado en la literatura (Tachet et al., 1980). La estación 6 se ha incluido en el grupo "No contaminados-Permanentes" para facilitar el análisis.

Las estaciones agrupadas dentro de la categoría de "Muy contaminadas" o "Contaminacion moderada" (Figura 4), presentaban un mayor porcentaje de organismos filtradores, especialmente Simúlidos o Quironomidos. En cambio, en las estaciones categorizadas dentro del grupo de "No contaminados-Permanentes" o de "No contaminadosTemporales" fueron muy abundantes los trituradores (Gammáridos, en su mayor parte), debido a que se trata de localidades situadas dentro del Parque con abundante vegetación de ribera que aporta material alóctono. Este tipo de organismos fueron poco abundantes en alguna de las estaciones fuera del Parque, donde aparecian como importantes los ramoneadores, como consecuencia del desarrollo de la comunidad algal.

En general se observa (Fig 4) que las localidades no contaminadas y con flujo relativamente alto (grupo "No contaminados-Permanentes")

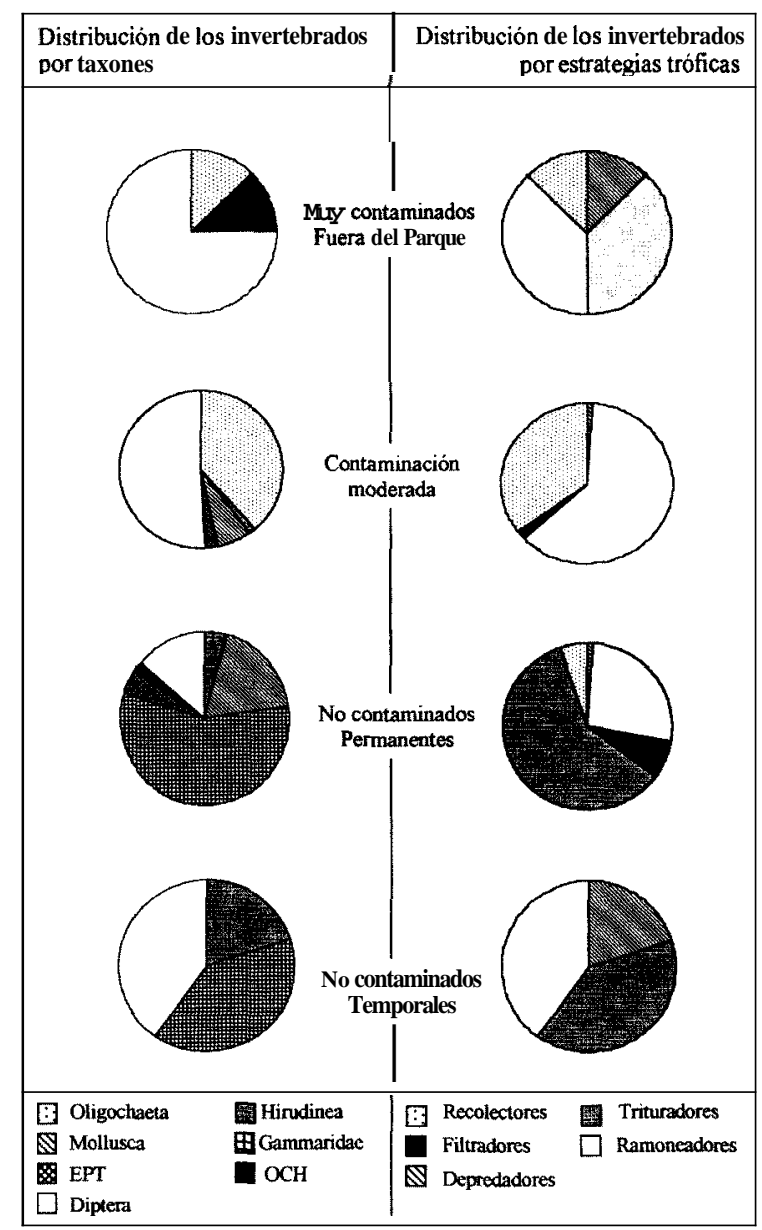

Figura 4. Representación de las abundancias relativas de los macroinvertebrados segdn grupos taxonómicos (izquierda) y grupos tróficos (derecha) en cada una de las comunidades definidas segdn el dendograma construido sobre una matriz de similaridades (Figura 2). EPT agrupa Efemerópteros, Plecopteros y Tricópteros, mientras que OCH agrupa Odonatos, Coleópteros y Heteropteros. Representation of relative abundances of different taxonomic groups of macroinvertebrates (left) and trophic strategies (right) in each of the mucroinvertehrute communities defined by the cluster analysis (Figure 2). EPT groups Efemeroptera, Plecoptera and Trichoptera, while OCH group Odonata, Coleopteru and Heteroptera.

poseian una comunidad más diversa y de estructura trófica más compleja comparadas con las localidades contaminadas y las no contaminadas con flujo bajo. 


\section{DISCUSIÓN}

Los rios mediterraneos cuentan con diversos estudios en Espaiia que han caracterizado sus comunidades y la relación de éstas con la contaminacion (Prat et al., 1985: Prat et al., 1985; Vidal-Abarca et al., 1991; Puig et al., 1986), ademas de otros trabajos que han estudiado grupos taxonómicos de forma particular (Suarez et al., 1986; Moreno et al., 1997), ciclos biologicos (Recasens, 1984; Recasens \& Puig, 1987; Ferreras-Romero \& García-Rojas, 1995) o la recolonización después de las riadas (Ortega et al., 1991). En estos estudios se destaca la relativa pobreza de estos rios en especies de Efemeropteros, Plecópteros y Tricopteros (rios EPT) y la abundancia de Colebpteros, Odonatos o Hemipteros (rios OCH) (Legier \& Talin, 1973; Alba-Tercedor et al., 1992; Malmqvist et al., 1993). Este patrón resulta muy marcado en las ramblas y rios del sudeste espaiiol (Moreno et al., 1996), aunque no lo es tanto cuando se estudian los rios de la zona de Cataluiia (Prat et al., 1985).

Los datos recolectados en la riera de Sant $\mathrm{Cu}$ gat nos hacian pensar a priori en un ecosistema muy pobre en especies, dada su proximidad a las grandes zonas urbanas cercanas a Barcelona donde se hace un uso intensivo y extensivo del agua. Sin embargo, la presencia del Parque Metropolitano de Collserola, permite que se mantengan comunidades de macroinvertebrados caracteristicos de aguas limpias, mas o menos complejas en función del grado de temporalidad del cauce. De entre las estaciones que se muestrearon en la cuenca de la Riera de Sant Cugat, tenemos localidades sin flujo dentro y fuera de la zona protegida, localidades con flujo reducido dentro del Parque y estaciones con flujo relativamente importante dentro y fuera de los limites del Parque. Ello permite comparar los efectos de la temporalidad y la contaminación sobre la comunidad de macroinvertebrados.

Las estaciones se agruparon en función de estas dos perturbaciones, encontrando los siguientes grupos de ordenación: estaciones "Muy contaminadas-Fuera del Parque", con "Contaminación moderada", "No contaminados-Perma- nentes" y "No contaminados-Temporales", estos dos ultimos formados enteramente con localidades situadas dentro del Parque. Los parametros que establecen las diferencias entre localidades situadas dentro o fuera de la zona protegida son: La riqueza taxonómica, la estructura trófica de la comunidad de macroinvertebrados, la calidad de las aguas y el desarrollo de la vegetación riparia. Las localidades externas al Parque presentaban un bosque de ribera bastante degradado, valores bajos de los indices biológicos aplicados y comunidades de macroinvertebrados muy sencillas tróficamente, 10 que en global implicaba una mala calidad del sistema. De las estaciones protegidas por el Parque, aquellas con mayor flujo tenian comunidades más complejas tanto en riqueza taxonomica como en estructura trofica. En cambio, aquellas con flujo reducido, tenian comunidades sencillas pues el poco caudal circulante no permitia mantener una compleja comunidad de macroinvertebrados. En cuanto a calidad de aguas, si bien en general las localidades dentro del Parque tenian muy buena calidad, el indice BMWP' indicaba que las localidades con flujo reducido tenian mala calidad a pesar de que los organismos encontrados son propios de aguas limpias. Esta dificultad en la aplicación del indice en los rios mediterraneos temporales también ha sido percibida en otros trabajos (Martínez-López et al., 1996; Coimbra et al., 1996: Dominguez et al., 1997), puesto que en todos los casos, el reducido numero de taxones disminuye el valor de este indice.

Tal y como se indica en otros trabajos realizados en zonas protegidas catalanas (Rieradevall $e t$ al., en prensa), se encuentran grandes abundancias de organismos trituradores y pocos ramoneadores, como consecuencia de que estas zonas gozan de una importante vegetación de ribera que aporta material e impide el desarrollo de comunidades algales importantes. En cambio en las zonas no protegidas se hallan comunidades de macroinvertebrados con abundantes organismos filtradores ya que existe una importante cantidad de material en suspension producto de la contaminación (Del Moral et al., 1997) y ramoneadores debido al gran desarrollo algal de algunas zonas. 
Asi pues, a pesar de que las crecidas y la temporalidad actúan como peturbaciones que podrían reducir de una forma importante la biodiversidad (Resh et al., 1990), y ello se da de forma importante en los rios mediterráneos (Maamri et al., 1997), ésta puede ser relativamenteelevada e incluso similar a la hallada en rios más pennanentes (Legier \& Talin, 1973; Boulton \& Suter, 1986; Boulton \& Lake, 1992) siempre que se mantenga un flujo permanente (aunque reducido) y/o zonas encharcadas con bosques de ribera bien conservados.

Todo ello puede verse en el estudio de la riera de Sant Cugat, donde el mosaico de condiciones dentro y fuera del parque y la presencia de pequeños torrentes, originan una comunidad más compleja de 10 que se puediera pensar "a priori" y que se puede constatar en un estudio extensivo en una sola época del año.

Nuestros datos muestran que las zonas protegidas tienen un potencial importante para que se desarrolle una fauna diversa, y que actuan como refugio de los macroinvertebrados caracteristicos de rios temporales mediterraneos no contaminados. También hay que destacar, que a pesar de ser rios con escaso caudal (y por tanto con poca dilución de la carga contaminante) presentan una considerable capacidad de autodepuración cuando ven alterada su calidad como ocurre en la transición del punto 9 al 6, 10 que también ha sido descrito en otros trabajos (Coimbra et al., 1996; Prenda \& Gallardo-Mayenco, 1996).

\section{AGRADECIMIENTO}

Este estudio se ha realizado gracias a la financiacion del proyecto CICYT AMB95-0220. Durante la realizacidn del presente trabajo, uno de los autores (N.B.) ha disfrutado de una beca FPI.

\section{BIBLIOGRAFÍA}

ALBA-TEKCERDOR, J. \& A. SÁNCHEZ-ORTEGA. 1988. Un método rapido y simple para evaluar la calidad biológica de las aguas corrientes basado en el de Helawell (1978). Limnetica, 4: 51-56.
ALBA-TERCEDOR, J., G. GONZÁLEZ \& M.A. PUIG. 1992. Present level of knowledge regarding fluvial macroinvertebrate communities in Spain. Limnetica, 8: 231-241.

BONADA N., M. RIERADEVALL \& N.PRAT. (2000). Biodiversitat i qualitat de l'aigua a la Riera de Sant Cugat (Collserola, Barcelona). I Jornades sobre la Recerca en els sistemes naturals de Collserola: aplicacions a la gestió del Parc: 45-49.

BOULTON, A. J. \& P.J. SUTER. 1986. Ecology of temporary streams- An Australian perspective. In: Limnology in Australia. P. De Deckker \& W. D. Williams (eds.): 313-327.

BOULTON, A.J. \& P.S. LAKE. 1992. The ecology of two intermittent streams in Victoria, Australia. II. Comparisons of faunal composition between habitats, rivers and years. Freshwat. Biol., 27: 99-121.

COIMBRA, C.N., M.A.S. GRAÇA \& R.M. CORTES. 1996. The effects of a basic effluent on macroinvertebrate community structure in a temporary mediterranean river. Environmental Pollution, 94(3): 301-307.

DEL MORAL, M., F. MARTÍNEZ-LÓPEZ \& A.M. PUJANTE. 1997. Estudio de los pequeños rios de la Sierra de Espadán (SO de Castellón). Macroinvertebrados y calidad de sus aguas. Ecologia, 11:37-61.

DOMÍNGUEZ, M. I., F. MARTINEZ-LOPEZ \& A. PUJANTE. 1997. Determinacidn de la calidad de las aguas en once arroyos de la cuenca del río $\mathrm{Ca}$ briel. Ecologia, 11: 63-81.

FEKRERAS-ROMERO, M. \& A.M. GARCÍA-ROJAS. 1995. Life-history and spatial separation exhibited by the odonates from a mediterranean inland catchment in southern Spain. Vie et Milieu, 45(2): 157-166.

GALLARDO, A. 1993. Macroinvertebrate associations in two basins of SW Spain. Arch. Hydrobiol. 127: 473-483.

GIUDICELLI, J., M. DAKKI \& A. DIA. 1985. Caractéristiques abiotiques et hydrobiologiques des eaux courantes méditerranéennes. Verh. Internat. Verein. Limnol., 22:2094-2101.

LEGENDKE, P. \& A. VAUDOR. 1991. The R Package: Multidimensional analysis, spatial analysis. Université de Montréal. 142 pag.

LEGIER, P. \& TALIN, J. 1973. Comparaison de ruisseaux permanents et temporaires de la provence calcaire. Annls. Limnol., 9(3): 273-292.

MAAMRI, A., H. CHERGUI \& E. PATTEE. 1997. Leaf litter processing in a temporary northeastern Moroccan river. Arch. Hydrobiol, 140(4): 513-531. 
MALMQVIST, B., A.N. NILSSON, M. BAEZ, P.D. ARMITAGE \& J. BLACKBURN. 1993. Stream macroinvertebrate communities in the island of Tenerife. Arch. Hydrobiol., 128(2): 209-235.

MARTÍNEZ-LÓPEZ, F., A. PUJANTE \& V. RIBARROCHE. 1996. Macroinvertebrados, comunidades vegetales y calidad de las aguas de la cuenca del río Palancia (Castellón, Valencia, Espaiia). Ecología, 10: 113-135.

MOLINA, C., M.R. VIDAL-ABARCA \& M.L. SUAREZ. 1994. Floods in arid southeast Spanish areas: a historical and environmental review. In: Coping with Floods. G. Rossi, N. Harmancioglu, V. Yevjevicc (eds.): 271-278.

MORENO, J.L., M.L. SUÁREZ \& M-R. VIDALABARCA. 1996. Valor ecológico de las ramblas como ecosistemas acuáticos singulares. Tomo Extraordinario. 125 Aniversario de la Real Sociedad Española de Historia Naatural: 411-415.

MORENO, J.L., A. MILLÁN, M.L. SUÁREZ, M.R. VIDAL-ABARCA \& J. VELASCO, 1997. Aquatic Coleoptera and Heteroptera assemblages in waterbodies from ephemeral coastal streams ("ramblas") of south.eastern Spain. Arch. Hydrobiol., 141(1): 93-107.

ORTEGA, M., M.L. SUAREZ, M.R. VIDAL-ABARCA, R. GÓMEZ \& L. RAMÍREZ-DÍAZ. 1991. Aspects of postflood recolonization of macroinvertebrates in a "Rambla" of South-East Spain ("Rambla del Moro": Segura River Basin). Verh. Internat. Verein.Limnol., 24: 1994-2001.

PRAT, N., G. GONZALEZ, X. MILLET \& M.A. PUIG. 1985. El Foix. Entre l'eixutesa i la contaminació. Monografies. Diputacid de Barcelona, 11, 95 pp.

PRAT, N., M. RIERADEVALL, A. MUNNÉ \& G. CHACON. 1996. La qualitat ecologica de les aigües del Besds i Llobregat. Estudis de la qualitat ecològica dels rius. Area de Medi Ambient. Diputació de Barcelona, 1. 102pp.

PRAT, N., M. RIERADEVALL, A. MUNNÉ, C. SOLA \& G. CHACON. 1997. La qualitat ecològica de les aigues del Besds i Llobregat. Estudis de la qualitat ecològica dels rius. Area de Medi Ambient. Diputacid de Barcelona, 2. 153 pp.

PRENDA, J. \& A. GALLARDO-MAYENCO. 1996. Self-purification, temporal variability and the macroinvertebrate community in small lowland Mediterranean streams receiving crude domestic sewage efluents. Arch. Hydrobiol., 136(2): 159-170.
PUIG, M.A., M. FERRERAS-ROMERO \& A.M.G. ROJAS. 1986. Ecosistemas de ríos temporales: Ecologia de las poblaciones de efemerópteros de la cuenca del río Bembézar (Sierra Morena). Anales de Biologia (BiologiaAmbiental). 8(2): 65-69.

RECASENS, L. 1984. Cicle vital d'Hydropsyche siltalai (Trichoptera: Hydropsychidae) a la riera de l'Avencó (Vallès Oriental). Bull. Inst. Cut. Hist. Nat., 52(6): 129-133.

RECASENS, L. \& M.A. PUIG. 1987. Life cycles and growth patterns of Trichoptera in the Matarraña, a karstic river. Proc. of the 5th Int. Symp. on Trichoptera. $247-251$.

RESH, V.H., J.K. JACKSON \& E.P. McELRAVY. 1990. Disturbance, annual variability, and lotic benthos: examples from a Californian stream influenced by a mediterranean climate. Mem. Inst. Ital. Idrobil., 47:309-329.

RIERADEVALL, M., N. BONADA \& N. PRAT. (en prensa). Community structure and water quality in the mediterranean streams of a natural parck (St. Llorenq del Munt, EN Spain). Limnetica.

SABATER, F., H. GUASCH, E. MARTÍ, J. ARMENGOL \& S. SABATER. 1995. The Ter: a mediterranean river case-study in Spain. Ecosystems of the world, 22. River and stream ecosystems. Cushing, C.E.; Cummins, K.W. \& Minshall, G.W (eds.): 419-438.

SABATER, S., F. SABATER \& J. ARMENGOL. 1993. Ecologia de los rios mediterraneos. Investigación y Ciencia. Agosto 1993: 72-79.

SUÁREZ, M.L., M. R. VIDAL-ABARCA, A.G. SOLER \& C. MONTES. 1986. Composición y estructura de una comunidad de larvas de odonatos (Zygoptera y Anisoptera) en un rio del SE de Espaiia: Cuenca del no Mula (rio Segura). Anales de Biología (Biología Ambiental), 8(2): 53-63

TACHET,H., M. BOURNAUD \& P. RICHOUX. 1980. Introduction à l'étude de macroinvertébrés des eaux douces (Systématique élémentaire et aperçu écologique). Ministère de l'Environnement. $156 \mathrm{pp}$.

VIDAL-ABARCA, M.R., M.L. SUAREZ, A. MILLÁN, R. GÓMEZ, M. ORTEGA, J. VELASCO, L. RAMÍREZ-DÍAZ. \& C. MONTES. 1991. Estudio limnoldgico de la cuenca del río Mundo (río Segura). Jornadas sobre el medio natural albacetense: $339-357$.

VIDAL-ABARCA, M. R., M.L. SUAREZ \& L. RAMÍREZ-DÍAZ. 1992. Ecology of Spanish semiarid streams. Limneticu, 8: 151-160. 